\title{
THE HAIGHT-RUZSA METHOD FOR SETS WITH MORE DIFFERENCES THAN MULTIPLE SUMS
}

\author{
MELVYN B. NATHANSON
}

\begin{abstract}
Let $h$ be a positive integer and let $\varepsilon>0$. The Haight-Ruzsa method produces a positive integer $m^{*}$ and a subset $A$ of the additive abelian group $\mathbf{Z} / m^{*} \mathbf{Z}$ such that the difference set is large in the sense that $A-A=$ $\mathbf{Z} / m^{*} \mathbf{Z}$ and $h$-fold sumset is small in the sense that $|h A|<\varepsilon m^{*}$. This note describes, and in a modest way extends, the Haight-Ruzsa argument, and constructs sets with more differences than multiple sums in other additive abelian groups.
\end{abstract}

\section{Sets With more sums than Differences}

Let $W$ be an additive abelian group. For every subset $A$ of $W$, we define the difference set

$$
A-A=\left\{a_{1}-a_{2}: a_{1}, a_{2} \in A\right\}
$$

and, for every positive integer $h$, the $h$-fold sumset

$$
h A=\left\{a_{1}+a_{2}+\cdots+a_{h}: a_{i} \in A \text { for all } i=1,2, \ldots, h\right\} .
$$

In particular,

$$
2 A=\left\{a_{1}+a_{2}: a_{1}, a_{2} \in A\right\}
$$

Because

$$
a_{1}+a_{2}=a_{2}+a_{1}
$$

but

$$
a_{1}-a_{2}=-\left(a_{2}-a_{1}\right)
$$

it is reasonable to expect that

$$
|A+A| \leq|A-A|
$$

for "most" but not necessarily all finite nonempty subsets $A$ of $W$. A set with more sums than differences is called an MSTD set. For example, in the additive group $\mathbf{Z}$ of integers, the set

$$
A=\{0,2,3,4,7,11,12,14\}
$$

is an MSTD set:

$$
\begin{gathered}
A+A=[0,28] \backslash\{1,20,27\} \\
A-A=[-14,14] \backslash\{ \pm 6, \pm 13\}
\end{gathered}
$$

and so

$$
|A+A|=26>25=|A-A| .
$$

Date: October 22, 2018.

2010 Mathematics Subject Classification. 05A17, 11B13, 11B30, 11B75, 11P99.

Key words and phrases. MSTD sets, additive number theory, combinatorial number theory.

Supported in part by a grant from the PSC-CUNY Research Award Program. 
Several families of MSTD sets of integers have been constructed, but there is no classification of such sets and many unsolved problems remain (cf. Hegarty [3], Hegarty and Miller [2, Iyer, Lazarov, Miller, and Zhang [4, 5], Martin and O'Bryant [6], Nathanson [7, 8).

A dual problem is to construct sets with more differences than multiple sums, that is, finite sets $A$ in a group $W$ such that the difference set $|A-A|$ is large but the $h$-fold sumset $h A$ is small. In 1973, Haight 1 proved that for all positive integers $h$ and $\ell$ there exists a modulus $m^{*}$ and a subset $A$ of $\mathbf{Z} / m^{*} \mathbf{Z}$ such that

$$
A-A=\mathbf{Z} / m^{*} \mathbf{Z}
$$

but $h A$ omits $\ell$ consecutive congruence classes. Recently, Ruzsa [9] refined and improved Haight's method, and proved the following: For every positive integer $h$ and every $\varepsilon>0$, there exists a modulus $m^{*}$ and a subset $A$ of $\mathbf{Z} / m^{*} \mathbf{Z}$ such that

$$
A-A=\mathbf{Z} / m^{*} \mathbf{Z}
$$

and

$$
|h A|<\varepsilon m^{*} .
$$

The purpose of this note is to describe, and in a modest way extend, the HaightRuzsa argument, and construct sets with more differences than multiple sums in other additive abelian groups.

\section{HAight-Ruzsa METHOD}

Let $W$ be an abelian group, and let $f: W \rightarrow W$ be a function, not necessarily a homomorphism. We define the subset

$$
A(W, f)=\{w+f(w): w \in W\} \cup\{f(w)): w \in W\} .
$$

For all $w \in W$, we have

$$
w=(w+f(w))-f(w)
$$

and so $A(W, f)$ is a subtractive basis for $W$, that is, $A(W, f)$ satisfies the difference set identity

$$
A-A=W \text {. }
$$

For every positive integer $h$ and $\varepsilon>0$, the Haight-Ruzsa method constructs a finite abelian group $W$ and a function $f: W \rightarrow W$ such that the $h$-fold sumset of $A(W, f)$ is small in the sense that

$$
|h A(W, f)|<\varepsilon|W| .
$$

Let $z \in W$. For every positive integer $h$, the element $z$ is in the $h$-fold sumset $h A(W, f)$ if and only if there exist functions

$$
\alpha, \beta: W \rightarrow\{0,1,2, \ldots, h\}
$$

such that

$$
h=\sum_{w \in W}(\alpha(w)+\beta(w))
$$

and

$$
z=\sum_{w \in W}(\alpha(w)(w+f(w))+\beta(w) f(w)) .
$$

A pair of functions $(\alpha, \beta)$ that satisfies conditions (2) and (3) is called an admissible pair for $W$, and the element $z \in h A(W, f)$ defined by (4) is called the group 
element represented by the admissible pair $(\alpha, \beta)$. An element $z \in h A(W, f)$ can be represented by many different admissible pairs.

An admissible pair $(\alpha, \beta)$ has level $\ell$ if

$$
\ell=\sum_{\substack{w \in W \\ \alpha(w)+\beta(w) \geq 1}} 1 .
$$

Condition (3) implies that $\alpha(w)+\beta(w) \geq 1$ for some $w \in W$, and so

$$
\ell \in\{1,2, \ldots, h\} \text {. }
$$

If $z$ in $h A$ is represented by the pair $(\alpha, \beta)$, then the level of the pair counts the number of $w \in W$ such that at least one of the group elements $w+f(w)$ and $f(w)$ appears in the representation (4) of $z$.

Let $L_{\ell}(W, f)$ be the set of all $z \in W$ such that $z$ can be represented by an admissible pair of level at most $\ell$. We have

$$
L_{1}(W, f) \subseteq L_{2}(W, f) \subseteq \cdots \subseteq L_{h}(W, f)=h A(W, f) .
$$

The Haight-Ruzsa method inductively constructs a sequence of groups

$$
W_{1} \subseteq W_{2} \subseteq \cdots \subseteq W_{h}=W
$$

and functions $f_{i}: W_{i} \rightarrow W_{i}$ for $i=1, \ldots, h$ such that

$$
A\left(W_{1}, f_{1}\right) \subseteq A\left(W_{2}, f_{2}\right) \subseteq \cdots \subseteq A\left(W_{h}, f_{h}\right)
$$

and the sumset $h A\left(W_{h}, f_{h}\right)=L_{h}\left(W_{h}, f_{h}\right)$ satisfies inequality (1).

\section{Preliminary}

Let $h$ be a positive integer, and let $\mathcal{R}_{h}$ be the set of commutative rings with identity such that, if $R \in \mathcal{R}_{h}$, then $r$ is a unit in $R$ for all $r \in\{1,2, \ldots, h\}$. For example, if $p$ is a prime number and $p>h$, then $\mathbf{Z} / p \mathbf{Z} \in \mathcal{R}_{h}$. Let $R_{0}, R_{1}, \ldots, R_{n} \in \mathcal{R}_{h}$. The direct sum $\bigoplus_{i=0}^{n} R_{i}$ is a ring with identity $\left(1_{R_{0}}, 1_{R_{1}}, \ldots, 1_{R_{n}}\right)$, and $\left(r_{0}, r_{1}, \ldots, r_{n}\right)$ is a unit in $\bigoplus_{i=0}^{n} R_{i}$ if $r_{i} \in\{1, \ldots, h\}$ for all $i=0,1, \ldots, n$. Thus, $\bigoplus_{i=0}^{n} R_{i} \in \mathcal{R}_{h}$. If $M_{i}$ is a finite $R_{i}$-module for $i=0,1, \ldots, n$, then $\bigoplus_{i=0}^{n} M_{i}$ is a finite $\bigoplus_{i=0}^{n} R_{i^{-}}$ module. The group $W_{h}$ will be constructed as the direct sum of a finite number of finite $R_{i}$-modules $M_{i}$, where $R_{i} \in \mathcal{R}_{h}$ for $i=0,1, \ldots, n$.

We use the following simple combinatorial inequality.

Lemma 1. Let $M_{0}, M_{1}, \ldots, M_{n}$ be finite sets, let

$$
W=M_{0} \times M_{1} \times \cdots \times M_{n}
$$

and let

$$
\left(x_{0}^{*}, x_{1}^{*}, \ldots, x_{n}^{*}\right) \in W
$$

If

$$
S=\left\{\left(x_{0}, x_{1}, \ldots, x_{n}\right) \in W: x_{j}=x_{j}^{*} \text { for some } j \in\{0,1, \ldots, n\}\right\}
$$

then

$$
|S| \leq|W| \sum_{j=0}^{n} \frac{1}{\left|M_{j}\right|}
$$

If $\varepsilon>0$ and $\left|M_{j}\right|>(n+1) / \varepsilon$ for all $j \in\{0,1, \ldots, n\}$, then $|S|<\varepsilon|W|$. 
Proof. The cardinality of $W$ is

$$
|W|=\prod_{i=0}^{n}\left|M_{i}\right| .
$$

For $j \in\{0,1, \ldots, n\}$, the number of elements $\left(x_{0}, x_{1}, \ldots, x_{n}\right) \in W$ with $x_{j}=x_{j}^{*}$ is

$$
\prod_{\substack{i=0 \\ i \neq j}}^{n}\left|M_{i}\right|=\frac{|W|}{\left|M_{j}\right|} .
$$

If

$$
S=\left\{\left(x_{0}, x_{1}, \ldots, x_{n}\right) \in W: x_{j}=x_{j}^{*} \text { for some } j \in\{0,1, \ldots, n\}\right\}
$$

then

$$
|S| \leq \sum_{j=0}^{n} \frac{|W|}{\left|M_{j}\right|}=|W| \sum_{j=0}^{n} \frac{1}{\left|M_{j}\right|} .
$$

If $\varepsilon>0$ and $\left|M_{j}\right|>(n+1) / \varepsilon$ for all $j \in\{0,1, \ldots, n\}$, then $|S|<\varepsilon|W|$. This completes the proof.

\section{INITIAL STEP}

Let $h$ be a positive integer and let $\varepsilon>0$. Choose numbers $\varepsilon_{1}, \ldots, \varepsilon_{h}$ such that

$$
0<\varepsilon_{1}<\varepsilon_{2}<\cdots<\varepsilon_{h}<\varepsilon .
$$

For $i=0,1,2, \ldots, h$, let $R_{i} \in \mathcal{R}_{h}$, let $M_{i}$ be a finite $R_{i}$-module such that $\left|M_{i}\right|>$ $(h+1) / \varepsilon_{1}$, and let

$$
W_{1}=\bigoplus_{i=0}^{h} M_{i}
$$

We write the element $w_{1} \in W_{1}$ as an $(h+1)$-tuple

$$
w_{1}=\left(x_{0}, x_{1}, \ldots, x_{h}\right)
$$

where $x_{i} \in M_{i}$ for $i=0,1, \ldots, h$. Recall that $h$ is a unit in $R_{i}$. For $i=0,1, \ldots, h$, we define the function $g_{i}: W_{1} \rightarrow M_{i}$ by

$$
g_{i}\left(w_{1}\right)=-\frac{i}{h} x_{i}
$$

and we define the function $f_{1}: W_{1} \rightarrow W_{1}$ by

$$
\begin{aligned}
f_{1}\left(w_{1}\right) & =\left(g_{0}\left(x_{0}\right), g_{1}\left(x_{1}\right), \ldots, g_{h}\left(x_{h}\right)\right) \\
& =\left(0,-\frac{1}{h} x_{1}, \ldots,-\frac{i}{h} x_{i}, \ldots,-\frac{h-1}{h} x_{h-1},-x_{h}\right)
\end{aligned}
$$

Let

$$
A\left(W_{1}, f_{1}\right)=\left\{w_{1}+f_{1}\left(w_{1}\right): w_{1} \in W_{1}\right\} \cup\left\{f_{1}\left(w_{1}\right): w_{1} \in W_{1}\right\} .
$$

The level 1 set $L_{1}\left(W_{1}, f_{1}\right)$ is the set of all $x \in h A\left(W_{1}, f_{1}\right)$ of the form

$$
x=j\left(w_{1}+f_{1}\left(w_{1}\right)\right)+(h-j) f_{1}\left(w_{1}\right)=j w_{1}+h f_{1}\left(w_{1}\right)
$$

for some

$$
j \in\{0,1,2, \ldots, h\} .
$$


We have

$$
\begin{aligned}
j w_{1} & +h f_{1}\left(w_{1}\right) \\
& =j\left(x_{0}, x_{1}, \ldots, x_{h}\right)+h\left(0,-\frac{1}{h} x_{1}, \ldots,-\frac{i}{h} x_{i}, \ldots,-\frac{h-1}{h} x_{h-1},-x_{h}\right) \\
& =\left(j x_{0}, j x_{1}, \ldots, j x_{i}, \ldots, j x_{h}\right)-\left(0, x_{1}, \ldots, i x_{i}, \ldots, h x_{h}\right) \\
& =\left(j x_{0},(j-1) x_{1}, \ldots,(j-i) x_{i}, \ldots,(j-h) x_{h}\right) .
\end{aligned}
$$

It follows from (17) that at least one coordinate of this $(h+1)$-tuple is 0. Applying Lemma 1, we obtain

$$
\left|L_{1}\left(W_{1}, f_{1}\right)\right| \leq\left|W_{1}\right| \sum_{i=0}^{h} \frac{1}{\left|M_{i}\right|}<\varepsilon_{1}\left|W_{1}\right| .
$$

\section{INDUCTIVE STEP}

Let $1 \leq k \leq h-1$, and assume that we have a ring $R_{0} \in \mathcal{R}_{h}$, a finite $R_{0}$-module $W_{k}$, and a function $f_{k}: W_{k} \rightarrow W_{k}$ such that the sets

$$
A\left(W_{k}, f_{k}\right)=\left\{w_{k}+f_{k}\left(w_{k}\right): w_{k} \in W_{k}\right\} \cup\left\{f_{k}\left(w_{k}\right): w_{k} \in W_{k}\right\}
$$

and

$$
L_{k}\left(W_{k}, f_{k}\right)=\left\{w_{k} \in h A_{k}: \operatorname{level}\left(w_{k}\right) \leq k\right\}
$$

satisfy

$$
\left|L_{k}\left(W_{k}, f_{k}\right)\right|<\varepsilon_{k}\left|W_{k}\right| .
$$

Because $W_{k}$ is a finite set, the number of admissible pairs on $W_{k}$ is finite. Let $n$ be the number of admissible pairs of level exactly $k+1$ with respect to $W_{k}$. We denote these pairs by $\left(\alpha_{i}, \beta_{i}\right)$ for $i=1,2, \ldots, n$. It follows from (3) that

$$
\alpha_{i}\left(w_{k}\right)+\beta_{i}\left(w_{k}\right) \in\{0,1, \ldots, h\}
$$

for all $w_{k} \in W_{k}$.

For $i=1,2, \ldots, n$, let $R_{i} \in \mathcal{R}_{h}$, and let $M_{i}$ be a finite $R_{i}$-module such that

$$
\left|M_{i}\right|>\frac{n}{\varepsilon_{k+1}-\varepsilon_{k}} \text {. }
$$

The set

$$
W_{k+1}=W_{k} \oplus \bigoplus_{i=1}^{n} M_{i}
$$

is a finite module over the ring $R_{0} \oplus \bigoplus_{i=1}^{n} R_{i} \in \mathcal{R}_{h}$. We denote the components of $w_{k+1} \in W_{k+1}$ as follows:

$$
w_{k+1}=\left(w_{k}, x_{1}, \ldots, x_{n}\right)
$$

where $w_{k} \in W_{k}$ and $x_{i} \in M_{i}$ for $i=1, \ldots, n$. Define the projection $\pi_{0}: W_{k+1} \rightarrow W_{k}$ by

$$
\pi_{0}\left(w_{k+1}\right)=w_{k}
$$

For $i=1,2, \ldots, n$, we define the projection $\pi_{i}: W_{k+1} \rightarrow M_{i}$ by

$$
\pi_{i}\left(w_{k+1}\right)=x_{i}
$$

and we define the function $g_{i}: W_{k+1} \rightarrow M_{i}$ as follows: If $\pi_{0}\left(w_{k+1}\right)=w_{k}$ and

$$
\alpha_{i}\left(w_{k}\right)+\beta_{i}\left(w_{k}\right)=0
$$


then

$$
g_{i}\left(w_{k+1}\right)=0 .
$$

If

$$
\alpha_{i}\left(w_{k}\right)+\beta_{i}\left(w_{k}\right) \in\{1, \ldots, h\}
$$

then

$$
g_{i}\left(w_{k+1}\right)=-\frac{\alpha_{i}\left(w_{k}\right) x_{i}}{\alpha_{i}\left(w_{k}\right)+\beta_{i}\left(w_{k}\right)} .
$$

The function $g_{i}$ is well defined because $r$ is a unit in $R_{i}$ for all $r \in\{1, \ldots, h\}$.

Define the function $f_{k+1}: W_{k+1} \rightarrow W_{k+1}$ by

$$
f_{k+1}\left(w_{k+1}\right)=\left(f_{k}\left(w_{k}\right), g_{1}\left(w_{k+1}\right), \ldots, g_{n}\left(w_{k+1}\right)\right) .
$$

We have

$$
w_{k+1}+f_{k+1}\left(w_{k+1}\right)=\left(w_{k}+f\left(w_{k}\right), x_{1}+g_{1}\left(w_{k+1}\right), \ldots, x_{n}+g_{n}\left(w_{k+1}\right)\right) .
$$

Consider the set

$$
\begin{aligned}
& A\left(W_{k+1}, f_{k+1}\right) \\
& \quad=\left\{w_{k+1}+f_{k+1}\left(w_{k+1}\right): w_{k+1} \in W_{k+1}\right\} \cup\left\{f_{k+1}\left(w_{k+1}\right): w_{k+1} \in W_{k+1}\right\} .
\end{aligned}
$$

Because

$$
\pi_{0}\left(f_{k+1}\left(w_{k+1}\right)\right)=f\left(w_{k}\right)
$$

and

$$
\pi_{0}\left(w_{k+1}+f_{k+1}\left(w_{k+1}\right)\right)=w_{k}+f\left(w_{k}\right)
$$

it follows that

$$
\pi_{0}\left(A_{k+1}\left(W_{k+1}, f_{k+1}\right)\right)=A_{k}\left(W_{k}, f_{k}\right) .
$$

The $(k+1)$-level set $L_{k+1}\left(W_{k+1}, f_{k+1}\right)$ is the set of all elements $z_{k+1} \in h A_{k+1}$ that can be represented by an admissible pair $(\gamma, \delta)$ of level at most $k+1$. We define the functions

$$
\hat{\gamma}: W_{k} \rightarrow \mathbf{N}_{0} \quad \text { and } \quad \hat{\delta}: W_{k} \rightarrow \mathbf{N}_{0}
$$

as follows: For $w_{k} \in W_{k}$, let

$$
\hat{\gamma}\left(w_{k}\right)=\sum_{\substack{\left(x_{1}, \ldots, x_{n}\right) \\ \in M_{1} \times \cdots \times M_{n}}} \gamma\left(w_{k}, x_{1}, \ldots, x_{n}\right)
$$

and

$$
\hat{\delta}\left(w_{k}\right)=\sum_{\substack{\left(x_{1}, \ldots, x_{n}\right) \\ \in M_{1} \times \cdots \times M_{n}}} \delta\left(w_{k}, x_{1}, \ldots, x_{n}\right)
$$

We have

$$
\hat{\gamma}\left(w_{k}\right)+\hat{\delta}\left(w_{k}\right)=\sum_{\substack{\left(x_{1}, \ldots, x_{n}\right) \\ \in M_{1} \times \cdots \times M_{n}}}\left(\gamma\left(w_{k}, x_{1}, \ldots, x_{n}\right)+\delta\left(w_{k}, x_{1}, \ldots, x_{n}\right)\right)
$$


and

$$
\begin{aligned}
\sum_{w_{k} \in W_{k}} & \left(\hat{\gamma}\left(w_{k}\right)+\hat{\delta}\left(w_{k}\right)\right) \\
& =\sum_{w_{k} \in W_{k}} \sum_{\substack{\left(x_{1}, \ldots, x_{n}\right) \\
\in M_{1} \times \cdots \times M_{n}}}\left(\gamma\left(w_{k}, x_{1}, \ldots, x_{n}\right)+\delta\left(w_{k}, x_{1}, \ldots, x_{n}\right)\right) \\
& =\sum_{w_{k+1} \in W_{k+1}}\left(\gamma\left(w_{k+1}\right)+\delta\left(w_{k+1}\right)\right) \\
& =h .
\end{aligned}
$$

Thus, $(\hat{\gamma}, \hat{\delta})$ is an admissible pair of functions on $W_{k}$.

Because $(\gamma, \delta)$ is an admissible pair of functions on $W_{k+1}$ of level at most $k+1$, it follows that $\gamma\left(w_{k+1}\right)+\delta\left(w_{k+1}\right) \geq 1$ for at most $k+1$ elements $w_{k+1} \in W_{k+1}$. Identity (8) implies that if $w_{k} \in W_{k}$ and $\hat{\gamma}\left(w_{k}\right)+\hat{\delta}\left(w_{k}\right) \geq 1$, then there exists $w_{k+1} \in W_{k+1}$ such that $\pi_{0}\left(w_{k+1}\right)=w_{k}$ and $\gamma\left(w_{k+1}\right)+\delta\left(w_{k+1}\right) \geq 1$. It follows that $\hat{\gamma}\left(w_{k}\right)+\hat{\delta}\left(w_{k}\right) \geq 1$ for at most $k+1$ elements $w_{k} \in W_{k}$, and so the pair $(\hat{\gamma}, \hat{\delta})$ has level at most $k+1$. Similarly, if the pair $(\gamma, \delta)$ has level at most $k$, then the pair $(\hat{\gamma}, \hat{\delta})$ has level at most $k$.

If $z_{k+1} \in L\left(W_{k+1}, f_{k+1}\right)$, then $z_{k+1}$ is represented by an admissible pair $(\gamma, \delta)$ of level at most $k+1$. We have

$$
\begin{aligned}
z_{k+1}= & \sum_{w_{k+1} \in W_{k+1}}\left(\gamma\left(w_{k+1}\right)\left(w_{k+1}+f_{k+1}\left(w_{k+1}\right)\right)+\delta\left(w_{k+1}\right) f_{k+1}\left(w_{k+1}\right)\right) \\
= & \sum_{w_{k+1} \in W_{k+1}}\left(\left(\gamma\left(w_{k+1}\right)\left(w_{k}+f\left(w_{k}\right), x_{1}+g_{1}\left(w_{k+1}\right), \ldots, x_{n}+g_{n}\left(w_{k+1}\right)\right)\right.\right. \\
& \left.\quad+\delta\left(w_{k+1}\right)\left(f\left(w_{k}\right), g_{1}\left(w_{k+1}\right), \ldots, g_{n}\left(w_{k+1}\right)\right)\right) .
\end{aligned}
$$

For $i=1, \ldots, n$, we have

$$
\begin{aligned}
\pi_{i}\left(z_{k+1}\right) & =\sum_{w_{k+1} \in W_{k+1}}\left(\gamma\left(w_{k+1}\right)\left(x_{i}+g_{i}\left(w_{k+1}\right)+\delta\left(w_{k+1}\right) g_{i}\left(w_{k+1}\right)\right)\right. \\
& =\sum_{w_{k+1} \in W_{k+1}}\left(\gamma\left(w_{k+1}\right) x_{i}+\left(\gamma\left(w_{k+1}\right)+\delta\left(w_{k+1}\right)\right) g_{i}\left(w_{k+1}\right)\right) \in M_{i} .
\end{aligned}
$$

For $i=0$, we have

$$
\begin{aligned}
z_{k} & =\pi_{0}\left(z_{k+1}\right) \\
& =\sum_{w_{k+1} \in W_{k+1}}\left(\gamma\left(w_{k+1}\right)\left(w_{k}+f\left(w_{k}\right)\right)+\delta\left(w_{k+1}\right) f\left(w_{k}\right)\right) \\
& =\sum_{w_{k} \in W_{k}} \sum_{\substack{\left(x_{1}, \ldots, x_{n}\right) \\
\in M_{1} \times \cdots \times M_{n}}}\left(\gamma\left(w_{k}, x_{1}, \ldots, x_{n}\right)\left(w_{k}+f\left(w_{k}\right)\right)+\delta\left(w_{k}, x_{1}, \ldots, x_{n}\right) f\left(w_{k}\right)\right) \\
& =\sum_{w_{k} \in W_{k}} \hat{\gamma}\left(w_{k}\right)\left(w_{k}+f\left(w_{k}\right)\right)+\hat{\delta}\left(w_{k}\right) f\left(w_{k}\right)
\end{aligned}
$$

and so $z_{k}$ is an element of $h A\left(W_{k}, f_{k}\right)$ that is represented by the admissible pair $(\hat{\gamma}, \hat{\delta})$ of level at most $k+1$. If the admissible pair $(\hat{\gamma}, \hat{\delta})$ has level at most $k$, then $z_{k} \in L_{k}\left(W_{k}, f_{k}\right)$. 
Suppose that $(\hat{\gamma}, \hat{\delta})$ is an admissible pair in $W_{k}$ of level exactly $k+1$. It follows that the admissible pair $(\gamma, \delta)$ in $W_{k+1}$ also has level exactly $k+1$. Because $\left\{\left(\alpha_{i}, \beta_{i}\right): i=1, \ldots, n\right\}$ is the set of all admissible pairs on $W_{k}$ of level $k+1$, there is a unique integer $j \in\{1,2, \ldots, n\}$ such that $(\hat{\gamma}, \hat{\delta})=\left(\alpha_{j}, \beta_{j}\right)$. Let

$$
S=\left\{w_{k} \in W_{k}: \alpha_{j}\left(w_{k}\right)+\beta_{j}\left(w_{k}\right) \geq 1\right\} .
$$

There are exactly $k+1$ elements $w_{k} \in W_{k}$ that appear in the representation of $z_{k}$ associated with the admissible pair $(\alpha, \beta)$, and so $|S|=k+1$. If $w_{k} \in S$, then

$$
\begin{aligned}
1 & \leq \alpha_{j}\left(w_{k}\right)+\beta_{j}\left(w_{k}\right)=\hat{\gamma}\left(w_{k}\right)+\hat{\delta}\left(w_{k}\right) \\
& =\sum_{\substack{\left(x_{1}, \ldots, x_{n}\right) \\
\in M_{1} \times \cdots \times M_{n}}}\left(\gamma\left(w_{k}, x_{1}, \ldots, x_{n}\right)+\delta\left(w_{k}, x_{1}, \ldots, x_{n}\right)\right) .
\end{aligned}
$$

Because the admissible pair $(\gamma, \delta)$ in $W_{k+1}$ has level $k+1$, for each $w_{k} \in S$, there is a unique $n$-tuple $\left(y_{1}, \ldots, y_{n}\right) \in M_{1} \times \cdots \times M_{n}$ such that

$$
\begin{aligned}
\alpha_{j}\left(w_{k}\right)+\beta_{j}\left(w_{k}\right) & =\hat{\gamma}\left(w_{k}\right)+\hat{\delta}\left(w_{k}\right) \\
& =\gamma\left(w_{k}, y_{1}, \ldots, y_{n}\right)+\delta\left(w_{k}, y_{1}, \ldots, y_{n}\right) \\
& \geq 1
\end{aligned}
$$

and

$$
\gamma\left(w_{k}, x_{1}, \ldots, x_{n}\right)+\delta\left(w_{k}, x_{1}, \ldots, x_{n}\right)=0
$$

for all $\left(x_{1}, \ldots, x_{n}\right) \in M_{1} \times \cdots \times M_{n}$ with $\left(x_{1}, \ldots, x_{n}\right) \neq\left(y_{1}, \ldots, y_{n}\right)$. Therefore,

$$
\begin{aligned}
\pi_{j}\left(z_{k+1}\right)= & \sum_{w_{k+1} \in W_{k+1}}\left(\gamma\left(w_{k+1}\right) x_{j}+\left(\gamma\left(w_{k+1}\right)+\delta\left(w_{k+1}\right)\right) g_{j}\left(w_{k+1}\right)\right) \\
= & \sum_{w_{k} \in W_{k}} \sum_{\substack{\left(x_{1}, \ldots, x_{n}\right) \in \\
M_{1} \times \cdots \times M_{n}}} \gamma\left(w_{k}, x_{1}, \ldots, x_{n}\right) x_{j} \\
& +\sum_{w_{k} \in W_{k}} \sum_{\substack{\left(x_{1}, \ldots, x_{n}\right) \in \\
M_{1} \times \cdots \times M_{n}}}\left(\gamma\left(w_{k}, x_{1}, \ldots, x_{n}\right)+\delta\left(w_{k}, x_{1}, \ldots, x_{n}\right)\right) g_{j}\left(w_{k+1}\right) \\
= & \sum_{w_{k} \in W_{k}}\left(\gamma\left(w_{k}, y_{1}, \ldots, y_{n}\right) x_{j}+\left(\gamma\left(w_{k}, y_{1}, \ldots, y_{n}\right)+\delta\left(w_{k}, y_{1}, \ldots, y_{n}\right)\right) g_{j}\left(w_{k+1}\right)\right) \\
= & \sum_{w_{k} \in W_{k}}\left(\alpha_{j}\left(w_{k}\right) x_{j}+\left(\alpha_{j}\left(w_{k}\right)+\beta_{j}\left(w_{k}\right)\right) g_{j}\left(w_{k+1}\right)\right) \\
= & \sum_{w_{k} \in W_{k}}\left(\alpha_{j}\left(w_{k}\right) x_{j}+\left(\alpha_{j}\left(w_{k}\right)+\beta_{j}\left(w_{k}\right)\right)\left(-\frac{\alpha_{j}\left(w_{k}\right) x_{j}}{\alpha_{j}\left(w_{k}\right)+\beta_{j}\left(w_{k}\right)}\right)\right) \\
= & 0 .
\end{aligned}
$$

To summarize, if $z_{k+1} \in L_{k+1}\left(W_{k+1}, f_{k+1}\right)$, then $\pi_{0}\left(z_{k+1}\right) \in L_{k}\left(W_{k}, f_{k}\right)$ or $\pi_{j}\left(z_{k+1}\right)=$ 0 for some $j \in\{1, \ldots, n\}$. Because $\left|L_{k}\left(W_{k}, f_{k}\right)\right|<\varepsilon_{k}\left|W_{k}\right|$, the number of elements $z_{k+1} \in W_{k+1}$ with $\pi_{0}\left(z_{k+1}\right) \in L_{k}\left(W_{k}, f_{k}\right)$ is at most

$$
\varepsilon_{k}\left|W_{k}\right|\left|M_{1}\right| \cdots\left|M_{n}\right|=\varepsilon_{k}\left|W_{k+1}\right| .
$$


For $j \in\{1, \ldots, n\}$, the number of elements in $W_{k+1}$ with $\pi_{j}\left(z_{k+1}\right)=0$ is

$$
\left|W_{k}\right| \prod_{\substack{i=1 \\ i \neq j}}^{n}\left|M_{i}\right|=\frac{\left|W_{k+1}\right|}{\left|M_{j}\right|}<\left(\frac{\varepsilon_{k+1}-\varepsilon_{k}}{n}\right)\left|W_{k+1}\right|
$$

and so

$$
\mid L_{k+1}\left(W_{k+1}, f_{k+1}\left|<\varepsilon_{k}\right| W_{k+1}\left|+\sum_{i=1}^{n}\left(\frac{\varepsilon_{k+1}-\varepsilon_{k}}{n}\right)\right| W_{k+1}\left|=\varepsilon_{k+1}\right| W_{k+1} \mid .\right.
$$

This completes the induction. With $k=h$, we obtain

$$
h A\left(W_{h}, f_{h}\right)=L_{h}\left(W_{h}, f_{h}\right)<\varepsilon_{h}\left|W_{h}\right|<\varepsilon\left|W_{h}\right| .
$$

This completes the proof.

\section{EXAMPLES}

Example 1: Let $h$ be a positive integer and let $\varepsilon>0$. Let $\mathcal{M}_{h}$ be the set of positive integers $m$ such that every prime divisor of $m$ is greater than $h$. If $m \in \mathcal{M}_{h}$, then every integer $r \in\{1,2, \ldots, h\}$ is a unit in the $\operatorname{ring} \mathbf{Z} / m \mathbf{Z}$, and so $\mathbf{Z} / m \mathbf{Z} \in \mathcal{R}_{h}$ and $\mathbf{Z} / m \mathbf{Z}$ is a finite $\mathbf{Z} / m \mathbf{Z}$-module. In the Haight-Ruzsa construction, if we choose modules $M_{i}=\mathbf{Z} / m_{i} \mathbf{Z}$ with pairwise relatively prime moduli $m_{i} \in \mathcal{M}_{h}$, then we obtain a finite abelian group

$$
W_{h}=\bigoplus_{i \in I} \mathbf{Z} / m_{i} \mathbf{Z} \cong \mathbf{Z} / m^{*} \mathbf{Z}
$$

where

$$
m^{*}=\prod_{i \in I} m_{i}
$$

and a function $f_{h}: W_{h} \rightarrow W_{h}$ such that the set

$$
A=A\left(W_{h}, f_{h}\right)=\left\{w_{h}+f_{h}\left(w_{h}\right): w_{h} \in W_{h}\right\} \cup\left\{f_{h}\left(w_{h}\right): w_{h} \in W_{h}\right\}
$$

satisfies $A-A=W_{h}$ and $|h A|<\varepsilon\left|W_{h}\right|$. This is Ruzsa's result, with $m_{i}$ prime for all $i$ and $m^{*}$ square-free.

Example 2: Let $h$ be a positive integer and let $\varepsilon>0$. Let $\mathbf{F}_{q_{i}}$ be the finite field with $q_{i}=p_{i}^{k_{i}}$ and $p_{i}>h$. In the Haight-Ruzsa construction, we can choose modules $M_{i}$ that are finite-dimensional vector spaces over the field $\mathbf{F}_{q_{i}}$ of sufficiently large dimension. We obtain a finite abelian group $W_{h}$ that is a direct sum of vector spaces. If each of these vector spaces is a vector space over the same field $\mathbf{F}_{q}$, then $W_{h}$ is a vector space over $\mathbf{F}_{q}$.

Example 3: Let $h$ be a positive integer and let $\varepsilon>0$. Let $\mathbf{F}_{q}$ be the finite field with $q=p^{k}$ and $p>h$, and let $\mathbf{F}_{q}[t]$ be the vector space of polynomials with coefficients in $\mathbf{F}_{q}$. Let $d_{0}=0<d_{1}<d_{2}<\cdots$ be a sufficiently rapidly increasing sequence of integers. We choose finite subspaces of $\mathbf{F}_{q}[t]$ of the form

$$
\left\{\sum_{j=d_{i-1}}^{d_{i}-1} c_{j} t^{j}: c_{j} \in \mathbf{F}_{q}\right\}
$$

The Haight-Ruzsa method produces a subspace $W_{h}$ of the vector space $\mathbf{F}_{q}[t]$ consisting of all polynomials of degree less than $d_{h}$. 


\section{OPEN PROBLEMS}

1. Let $h$ be a positive integer and $\varepsilon>0$. Does there exist a prime $p$ and a set $A$ in $\mathbf{Z} / p \mathbf{Z}$ such that $A-A=\mathbf{Z} / p \mathbf{Z}$ and $|h A|<\varepsilon p$ ? Do such sets exist for all sufficiently large primes $p$ ?

2. Let $h$ be a positive integer, let $\varepsilon>0$, and let $c>0$. Do there exist an additive abelian group $W$ with $|W|>c$ and a subset $A$ of $W$ such that $A-A=W$ and the set

satisfies

$$
G=\left\{2 a_{1}+a_{2}+\cdots+a_{h}: a_{i} \in A \text { for all } i=1,2, \ldots, h\right\}
$$

$$
|G|<\varepsilon|W| ?
$$

3. Let $F\left(x_{1}, \ldots, x_{h}\right)=\sum_{i=1}^{h} r_{i} x_{i}$ be a linear form with coefficients in a ring $R$, and let $W$ be an $R$-module. For every subset $A$ of $W$, we define

$$
F(A)=\left\{\sum_{i=1}^{h} r_{i} a_{i}: a_{i} \in A \text { for all } i=1, \ldots, h\right\} .
$$

It is an open problem to determine the pairs of linear forms $(F, G)$ such that, for every $\varepsilon>0$ and $c>0$, there exists a finite $R$-module $W$ with $|W|>c, F(A)=W$, and $|G(A)|<\varepsilon|W|$. The Haight-Ruzsa produces modules for which this condition is satisfied for the pair of linear forms $(F, G)$, where

$$
F\left(x_{1}, x_{2}\right)=x_{1}-x_{2}
$$

and

$$
G\left(x_{1}, \ldots, x_{h}\right)=x_{1}+\cdots+x_{h}
$$

4. If $F$ and $G$ are polynomials with coefficients in a commutative ring $R$, and if $\varepsilon>0$ and $c>0$, does there exist a finite $R$-algebra $W$ with $|W|>c$ and a subset $A$ of $W$ such that $F(A)=W$ and $|G(A)|<\varepsilon|W|$.

\section{REFERENCES}

[1] J. A. Haight, Difference covers which have small $k$-sums for any $k$, Mathematika 20 (1973), 109-118.

[2] P. Hegarty and S. J. Miller, When almost all sets are difference dominated, Random Structures Algorithms 35 (2009), no. 1, 118-136.

[3] P. V. Hegarty, Some explicit constructions of sets with more sums than differences, Acta Arith. 130 (2007), 61-77.

[4] G. Iyer, O. Lazarev, S. J. Miller, and L. Zhang, Generalized more sums than differences sets, J. Number Theory 132 (2012), no. 5, 1054-1073.

[5] _ Finding and counting MSTD sets, Combinatorial and additive number theoryCANT 2011 and 2012, Springer Proc. Math. Stat., vol. 101, Springer, New York, 2014, pp. 7998.

[6] G. Martin and K. O'Bryant, Many sets have more sums than differences, Additive combinatorics, CRM Proc. Lecture Notes, vol. 43, Amer. Math. Soc., Providence, RI, 2007, pp. 287-305.

[7] M. B. Nathanson, Problems in additive number theory. I, Additive combinatorics, CRM Proc. Lecture Notes, vol. 43, Amer. Math. Soc., Providence, RI, 2007, pp. 263-270.

[8] _ Sets with more sums than differences, Integers 7 (2007), A5, 24.

[9] I. Z. Ruzsa, More differences than multiple sums, arXiv: 1601.04146, 2016.

Department of Mathematics, Lehman College (CUNY), Bronx, NY 10468

E-mail address: melvyn.nathanson@lehman.cuny.edu 